\title{
Coupling between lipid miscibility and phosphotyrosine driven protein condensation at the membrane
}

J. K. Chung, ${ }^{*}$ W. Y. C. Huang, ${ }^{*}$ C. B. Carbone, L. M. Nocka, A. N. Parikh, R. D. Vale, J. T. Groves $^{\#}$

${ }^{*}$ These authors contributed equally to this work.

${ }^{*}$ To whom correspondence should be addressed. 


\begin{abstract}
Lipid miscibility phase separation has long been considered to be a central element of cell membrane organization. More recently, protein condensation phase transitions, into threedimensional droplets or in two-dimensional lattices on membrane surfaces, have emerged as another important organizational principle within cells. Here, we reconstitute the LAT:Grb2:SOS protein condensation on the surface of giant unilamellar vesicles capable of undergoing lipid phase separations. Our results indicate that assembly of the protein condensate on the membrane surface can drive lipid phase separation. This phase transition occurs isothermally and is governed by tyrosine phosphorylation on LAT. Furthermore, we observe that the induced lipid phase separation drives localization of the SOS substrate, K-Ras, into the LAT:Grb2:SOS protein condensate.
\end{abstract}

\title{
Statement of Significance
}

Protein condensation phase transitions are emerging as an important organizing principles in cells. One such condensate plays a key role in $\mathrm{T}$ cell receptor signaling. Immediately after receptor activation, multivalent phosphorylation of the adaptor protein LAT at the plasma membrane leads to networked assembly of a number of signaling proteins into a two-dimensional condensate on the membrane surface. In this study, we demonstrate that LAT condensates in reconstituted vesicles are sufficient to drive lipid phase separation. This lipid reorganization drives another key downstream signaling molecule, Ras, into the LAT condensates. These results show that the LAT condensation phase transition, which is actively controlled by phosphorylation reactions, extends its influence to control lipid phase separation in the underlying membrane. 


\section{Introduction}

In 1973, shortly after the classic fluid mosaic description of cell membranes was published (1), a series of papers from Harden McConnell's lab described discovery of lateral phase separation in the lipids of cell membranes (2-6). Contemporary work from Erich Sackmann and colleagues confirmed an intriguing heterogeneity in the organization of lipids in the fluid membrane (7). This phenomenon later developed into the lipid raft model of cell membranes, as articulated by Kai Simons and Elina Ikonen in 1997 (8-10). The field of lipid rafts has since both flourished and attracted great controversy (11-14). Although lipid miscibility phase transitions are readily, and spectacularly visualized in purified lipid membranes (15-17), their unambiguous detection in living cells proved much more challenging (18-21), with only very limited definitive observations reported (22). There is evidence that cell membranes are poised near a miscibility phase transition (23), which naturally leads one to speculate that this may be actively controlled by the cell. However, a longstanding criticism of the lipid raft model questions how lipid phase separation could be controlled with the specificity required for biological functions, while the underlying interactions between lipids and cholesterol that enable the phase transition are rather nonspecific (24). Clearly proteins must play a commanding role controlling lipid phase separation in the physiological setting, but we have very limited mechanistic understanding of how this is actually achieved in specific cases (25).

One prominent example that captures this debate is the T-cell receptor (TCR) signaling system. TCR and a number of downstream proteins including linker for activation of T cells (LAT), phospholipase C gamma 1 (PLC $\gamma 1$ ), and the Ras activator son of sevenless (SOS), form clusters on the membrane (26-32). Earlier studies using detergent-resistant membrane (DRM) extraction 
have suggested that these molecules reside on lipid rafts (33-35). However, subsequent studies have failed to conclusively establish lipid rafts as the driving force for TCR-induced signaling clusters (36-38). Furthermore, it remains unclear how signaling activity - in the case of TCR, the receptor activation and tyrosine phosphorylation of downstream proteins including LAT_could trigger the lipid phase separation. This disconnect is further underscored by the fact that, at physiological ligand densities (39), individual TCR are capable of triggering the entire signaling pathway without ever forming clusters themselves (40-44).

Modular binding interactions among proteins present another type of mediated molecular assembly process in cells $(45,46)$. With sufficient multivalency, these interactions can lead to protein condensation phase transitions into three-dimensional droplets (47), sometimes called membraneless organelles, or two-dimensional assemblies on the membrane surface (48-51). Similar biomolecular condensates can also incorporate nucleic acids and play a role in transcription regulation $(52,53)$.

It has recently been discovered that LAT can participate in a protein condensation phase transition in reconstituted membranes $(48,49,54,55)$. LAT is a transmembrane scaffold protein that becomes phosphorylated at multiple tyrosines upon TCR activation. Three of the phosphotyrosines on LAT are canonical docking sites for the $\mathrm{SH} 2$ domain of growth factor receptor-bound protein 2 (Grb2), a cytosolic adapter protein (56). Grb2 additionally has SH3 domains, which bind to the proline-rich domain of SOS, a guanine nucleotide exchange factor that activates Ras (57). A single SOS can associate with at least two Grb2 molecules, and these multivalent interactions result in an extended two-dimensional network assembly of LAT:Grb2:SOS on the membrane in a 
phosphorylation-dependent manner $(58,59)$. This complex has been shown to play an important role in T-cell signaling $(60,61)$. The LAT:Grb2:SOS protein condensation phase transition is reversible, and since it is governed by tyrosine phosphorylation, it is directly under the control of competing kinase and phosphatase reactions in the TCR signaling system.

Here we reconstitute the LAT:Grb2:SOS protein condensate from purified proteins on giant unilamellar vesicle (GUV) membranes that can undergo lipid phase separation. The cytoplasmic domain of LAT was purified with an N-terminal His 6 tag and labeled with Alexa Fluor 555 (AF555) at Cys146 via maleimide-thiol chemistry. LAT was phosphorylated by the kinase domain of Hck in solution. Then, phosphorylated LAT ( $p$ LAT) was linked to the membrane by the binding of His 6 tag to the Ni-NTA lipids in the membrane. This membrane-linked $p$ LAT exhibits free lateral diffusion and remains monomeric prior to any assembly $(48,49)$. The addition of full-length Grb2 and the proline-rich domains of SOS leads to the networked condensation of LAT:Grb2:SOS on the membrane surface of GUVs, as shown in Figure 2 (top row). Here, the condensates are visualized as concentrated regions of $p$ LAT-AF555 fluorescence on GUVs by confocal microscopy. This condensate is mediated by tyrosine phosphorylation on LAT, and is reversible (Figure 2, bottom row). The rapid $(<10 \mathrm{~s})$ dispersion of the condensed structure upon phosphatase $(Y o p H)$ addition indicates that the individual Grb2:pLAT bonds must be highly dynamic and offer little protection from solution phosphatases. Incidentally, the membrane-linked phosphatase CD45 has been reported to be excluded from LAT condensates, possibly providing some degree of positive feedback with respect to this phosphatase (48).

We next examined how the lipid phase transition behavior of GUVs is perturbed by the LAT condensate. GUVs composed of a ternary mixture of saturated lipids, unsaturated lipids, and 
sterols (in a roughly 1:1:1 ratio) exhibit temperature-dependent miscibility phase separation. Below the miscibility transition temperature, $T_{\text {misc}}$, the vesicles separate into coexisting liquid ordered $\left(L_{\mathrm{o}}\right)$ and liquid disordered $\left(L_{\mathrm{d}}\right)$ regions $(16,62)$. As a crude guideline, the $L_{\mathrm{o}}$ region is rich in saturated phosphatidylcholine (PC) lipids such as DPPC, while $L_{\mathrm{d}}$ is rich in unsaturated phospholipids such as DOPC (63). For our experiments, GUVs composed of 29.2\% DOPC, 33.2\% DPPC, 33.3\% cholesterol, 4\% Ni-DOGS, 0.1\% Texas Red-DHPE (TR-DHPE), and 0.2\% Oregon Green-DHPE (OG-DHPE) were used. This composition is an approximation of the wellcharacterized equimolar mixture of DOPC, DPPC, cholesterol, and the observed $T_{\text {misc }}$ is also close to the reported value, $29^{\circ} \mathrm{C}(62)$. Even though it is not critical into which lipid phase LAT partitions in our experiments, the full length protein has been shown to partition into clusters without lipid raft makers (GPI anchors) in live cells (38) - suggesting that LAT does not partition into $L_{0}$-like phase in cells. In our experiments, because Ni-DOGS chain is unsaturated (18:1-18:1), Nichelated $p$ LAT is expected partition into the $L_{\mathrm{d}}$ region. This is confirmed by its colocalization with TR-DHPE, which is a well-established reporter of $L_{\mathrm{d}}$ phase (16). On the other hand, TR and OG fluorescence exclude each other upon phase separation, indicating that OG-DHPE partitions into the $L_{\mathrm{o}}$ phase (Figure 1A).

First, we examined whether LAT condensation could induce phase transitions in initially uniform vesicles near the miscibility transition temperature. The experiment is shown in Figure 3. In the imaging chamber maintained at $31^{\circ} \mathrm{C}$, the $p$ LAT-associated vesicle membranes exhibit a homogeneous distribution of fluorescent markers (TR-DHPE and OG-DHPE), as expected since this temperature is slightly above the $T_{\text {misc }}$ of $29^{\circ} \mathrm{C}$. The addition of Grb2-AF647 and SOS triggers a rapid LAT:Grb2:SOS condensation on the membrane surface, which is readily visualized by the appearance of concentrated regions of $647 \mathrm{~nm}$ fluorescence, tracking Grb2. This is accompanied 
by a clear partitioning of TR-DHPE (yellow) and OG-DHPE (blue), indicating a miscibility phase transition within the lipids has also occurred, although here under isothermal conditions. The LAT condensate is coincident with the $L_{\mathrm{o}}$ region marked by TR-DHPE, while $L_{\mathrm{d}}$ region, visualized by OG-DHPE, is excluded from LAT.

Next, we examined the temperature-dependent phase separation behavior of the vesicles in equilibrium under different conditions (Figure 4A). In this experiment, the temperature of the imaging chamber was increased gradually, at a rate of about $1^{\circ} \mathrm{C} / \mathrm{min}$, and held at each temperature data point for $2 \mathrm{~min}$. Then, multichannel confocal images of a population $(n \sim 100)$ of vesicles were obtained. The chamber was cooled back to $20^{\circ} \mathrm{C}$ at the same rate. The number of phase-separated vesicles were counted at each temperature point. All observations were the same regardless of the direction of the temperature ramp, indicating that all processes, including protein assembly and lipid phase separation, are reversible. Bare GUVs (empty black circles) show $T_{\text {misc }}$ of $29^{\circ} \mathrm{C}$. With $p$ LAT associated with the vesicles (solid black circles), $T_{\text {misc }}$ is shifted slightly but remains essentially the same at $28^{\circ} \mathrm{C}$. When the LAT condensate is formed by the addition of Grb2 and SOS, however, the apparent $T_{\text {misc }}$ is increased to $34^{\circ} \mathrm{C}$ (red solid circles). This is consistent with the previous experiment in which the lipid phase separation is driven by the protein condensate at a temperature where it would otherwise be homogeneous.

The apparent $\Delta T_{\text {misc }}$ of $5^{\circ} \mathrm{C}$ in the presence of the protein condensate is not actually a shift in the lipid $T_{\text {misc. }}$ Rather, the protein condensate itself becomes unstable at higher temperatures, and Grb2 and SOS are released from the vesicle surfaces. This can be seen in Figure 4A, bottom right: at $39^{\circ} \mathrm{C}$, the Grb2-AF647 fluorescence is not redistributed on the membranes, but rather reduced overall because it was lost to the solution. The fluorescence signal is recovered when the temperature is lowered, indicating that the protein condensation is also a reversible, temperature- 
dependent process. As long as the protein condensate is present, vesicles remained phase-separated with the $L_{\mathrm{d}}$ region templating the protein condensate (Figure 4). This suggests that the actual $\Delta T_{\text {misc }}$ is greater than the apparent value of $5^{\circ} \mathrm{C}$, and probably lies outside the experimentally accessible temperature range where both GUV phase separation and LAT:Grb2:SOS condensate can be observed.

Finally, we investigated how the spatial organization of other membrane-bound proteins might be directed by the protein assembly-induced lipid phase separation. K-Ras is a small GTPase and a substrate of SOS, and SOS-catalyzed nucleotide exchange from its GDP- to its GTP-bound state triggers downstream signal activation. The various Ras isoforms serve as hubs for signaling pathways such as phosphoinositide 3-kinases (PI3K) and mitogen-activated protein kinase (MAPK), and Ras misregulation is among the most common causes of cancer $(64,65)$. Native KRas is localized to the membrane by a farnesyl lipid modification, as well as electrostatic interactions between its positively charged region and anionic phospholipids in cellular membranes (66). Therefore, the organization of lipids is expected to play an important role in determining the location of K-Ras. Previous studies have shown that K-Ras partitions to the $L_{\mathrm{d}}$ region on GUVs, largely due to the highly branched farnesyl anchor (67). Therefore we anticipated K-Ras may similarly be directed by the lipid phase separation induced by the LAT:Grb2:SOS condensate. To examine this, eGFP-labeled full-length K-Ras with its native membrane anchor, including both the farnesylation and methylation of the terminal cysteine $(68,69)(20 \mathrm{nM}$ final concentration), was introduced into GUVs of similar composition as in the previous experiments, but with 10\% anionic DOPS lipids (final composition: $19.3 \%$ DOPC, 33.3\% DPPC, 33.3\% cholesterol, 10\% DOPS, 4\% Ni-DOGS, 0.1\% TR-DHPE). The negatively charged lipids are necessary for the stable association of K-Ras to the membrane (69-71). The bottom panel of Figure 
5 shows that, prior the introduction of Grb2 and SOS, eGFP-K-Ras (blue) as well as TR-DHPE (yellow) are initially distributed homogeneously on the vesicles. After Grb2 (red) and SOS are added, the lipid membrane becomes phase-separated as the protein assemblies form on its surface. K-Ras, LAT:Grb2:SOS, and TR are observed to partition together in the $L_{\mathrm{d}}$ region. Coupling of the lipid miscibility phase separation to the LAT:Grb2:SOS protein condensation localizes K-Ras with the condensate. As K-Ras does not colocalize with the protein condensate on supported lipid bilayers that are incapable of phase transitions (Figure S1), its partitioning on GUVs is likely to be driven by its anchor participating in the lipid phase transition. This phase transition and subsequent protein colocalization between SOS and K-Ras occurs isothermally, and under the control of tyrosine phosphorylation reactions.

In summary, we have reconstituted the T-cell signaling condensate, LAT:Grb2:SOS, on vesicles capable of undergoing liquid-liquid miscibility phase transitions. We observed that the formation of protein condensate can drive the lipid phase transition under isothermal conditions, redistributing lipids in a signal-dependent manner. Furthermore, we have shown that K-Ras, which does not directly participate in the LAT:Grb2:SOS condensation, nonetheless colocalizes with the condensate through its sensitivity to the lipid environment. Lipid phase separation can also be induced by actin polymerization $(72,73)$ and lipid crosslinking by cholera toxin $(74)$. Unique to the observations reported here, however, is that the LAT:Grb2:SOS protein condensation occurs immediately downstream of TCR activation, and as a direct result of ZAP70 kinase activation on triggered TCRs $(28,75)$. ZAP70 is a Syk Family Kinase that exhibits a distinctive substrate specificity, orthogonal to that of other kinases in the TCR signaling system, and strongly favors phosphorylation of the specific tyrosine residues on LAT that are involved in the LAT condensate 
(76). In this way, the LAT condensation phase transition is selectively controlled by TCR signaling.

The native LAT protein has been reported to exhibit a similar preference for the $L_{\mathrm{d}}$ lipid phase as the lipid-linked LAT in our experiments (38). However, in light of the significant number of other membrane-associated and transmembrane proteins in the cellular context of the LAT signaling condensate (59), we would refrain from extrapolating these results to predict specific details of the lipid phase associated with LAT in the natural physiological setting. The important point is that LAT condensation perturbs the underlying lipids and is capable of inducing lipid phase separation, now under the control of TCR signaling (Fig. 5). The LAT:Grb2:SOS protein condensate is not unique. Other two-dimensional condensates have been discovered, with their own signaling specificities $(50,51)$, and more are likely to emerge (e.g. with EGFR, which shares multivalent Grb2 and SOS interactions much like LAT). Such protein condensates on the membrane may play a broad role directly connecting receptor signaling activity with membrane lipid phase structure.

From a more physical perspective, a distinctive feature of the coupled protein-membrane system is that it exhibits phase transitions isothermally, and under control of competitive kinasephosphatase reactions. At a single temperature and composition, the molecular interactions themselves change (as a function of LAT phosphorylation), and the phase state of the system follows. This differs from typical observations of lipid miscibility phase transitions, in which the molecular properties of the lipids are fixed, and other control parameters such as temperature potentiate the phase transition $(16,77-79)$. This control over LAT condensation through tyrosine phosphorylation not only enables the specific connection with cellular signaling systems, it also opens the door to various nonequilibrium chemical phenomena. 
An example for such nonequilibrium phenomena can be found in a competitive lipid kinase-phosphatase reaction, which is similar to the tyrosine kinase-phosphatase competition governing LAT phosphorylation. The lipid kinase-phosphatase system has recently been observed to exhibit scale sensitivity in which the final outcome of the reaction depends on the size of the reaction system (e.g. a corralled lipid membrane in micron scales) (80). In this case, under identical concentrations of lipid kinases and phosphatases in solution, the membrane reaction system reaches a $\mathrm{PIP}_{2-}$ or $\mathrm{PIP}_{1-}$ (lipid kinase and phosphatase products, respectively) dominated state based on size and degree of confinement by the corralled membranes. Even partially confined membrane features, such as filopodia, are sufficient to flip the reaction outcome, and more elaborate pattern formations occur under different geometric restrictions. As with all kinasephosphatase competitive cycles, this example is a dissipative process that continuously consumes ATP. The system is intrinsically out of equilibrium and the mechanism of this reaction scale sensitivity is rooted in nonequilibrium aspects of the kinetic system (81). The tyrosine kinasephosphatase reactions upstream of the LAT condensate are qualitatively similar to the lipid kinasephosphatase system mentioned above, albeit with even more complex feedback and regulatory couplings $(82,83)$. In the case of the LAT condensate, functionally critical properties such as nucleation threshold, size distribution, and growth-dispersion characteristics, are likely to be set by the kinase-phosphatase reactions controlling LAT phosphorylation. The LAT condensates, as well as any lipid phase structure they cause, will thus reflect the chemical states of the signaling system - including those arising from nonequilibrium processes - rather than equilibrium phase separation. At the present time, very little is known about physical characteristics of LAT condensates in living cells, leaving a wealth of opportunities for detailed studies of these systems. From a functional perspective, one may speculate that lipid miscibility phase separation in living 
bioRxiv preprint doi: https://doi.org/10.1101/2020.07.22.215970; this version posted July 23, 2020. The copyright holder for this preprint (which was not certified by peer review) is the author/funder. All rights reserved. No reuse allowed without permission.

cells is inextricably coupled to numerous specific protein assemblies and signaling processes, many of which are only beginning to gain visibility. 


\section{Acknowledgments}

This work was supported by the Novo Nordisk Foundation Challenge Program under the Center for Geometrically Engineered Cellular Systems. Additional support was provided by National Institutes of Health (NIH) National Cancer Institute (NCI) Physical Sciences in Oncology Network (PS-ON) project 1-U01CA202241 and by NIH grant P01 A1091580. We thank John-Paul Denson, William K. Gillette, and Andrew G. Stephen (NCI RAS Initiative, Frederick National Laboratory

for Cancer Research) for the eGFP-K-Ras construct. We thank Young Kwang Lee, Hiu Yue Monatrice Lam, Shalini Low-Nam, and Emily C. Laubscher for assistance with the pilot experiments.

\section{Author Contribution}

J. K. Chung, W. Y. C. Huang, and J. T. Groves conceived the research.

J. K. Chung, W. Y. C. Huang, C. B. Carbone, and L. M. Nocka performed experiments.

J. K. Chung, W. Y. C. Huang, C. B. Carbone, R. D. Vale, A. N. Parikh, and J. T. Groves analyzed data and interpreted results.

J. K. Chung, W. Y. C. Huang, and J. T. Groves wrote the manuscript.

All authors commented on the manuscript. 


\section{Figures}

A
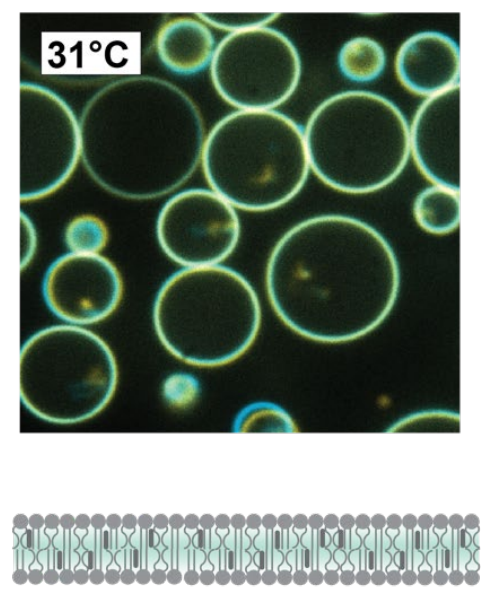

B

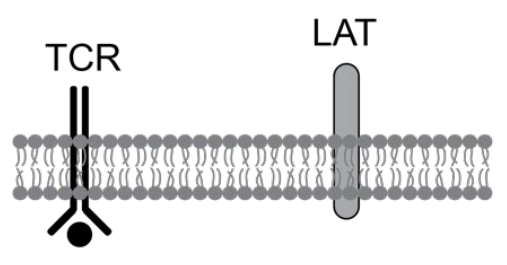

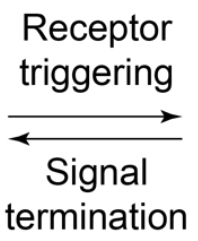
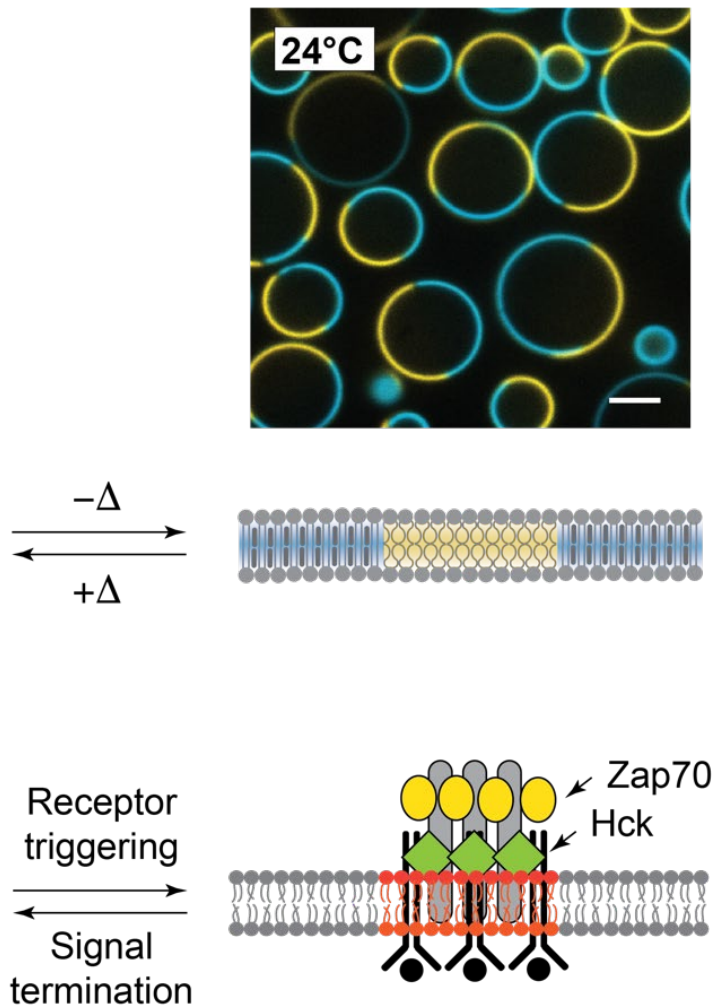

Figure 1. (A) Temperature-dependent liquid-liquid phase separation in giant unilamellar vesicles (GUVs). Above the transition temperature $T_{\text {misc, }}$ the distribution of lipids is homogeneous across the membrane (left). Below $T_{\text {misc }}$, lipids compartmentalize into macroscopic domains: the $L_{\mathrm{d}}$ domain (yellow, TR-DHPE) is enriched with unsaturated lipids, and the $L_{\mathrm{o}}$ with saturated lipids (blue, OG-DHPE). (B) In lipid raft theory, clusters of signaling proteins such as the T-cell receptors, are "carried" on ordered lipid domains to facilitate signal transduction. 


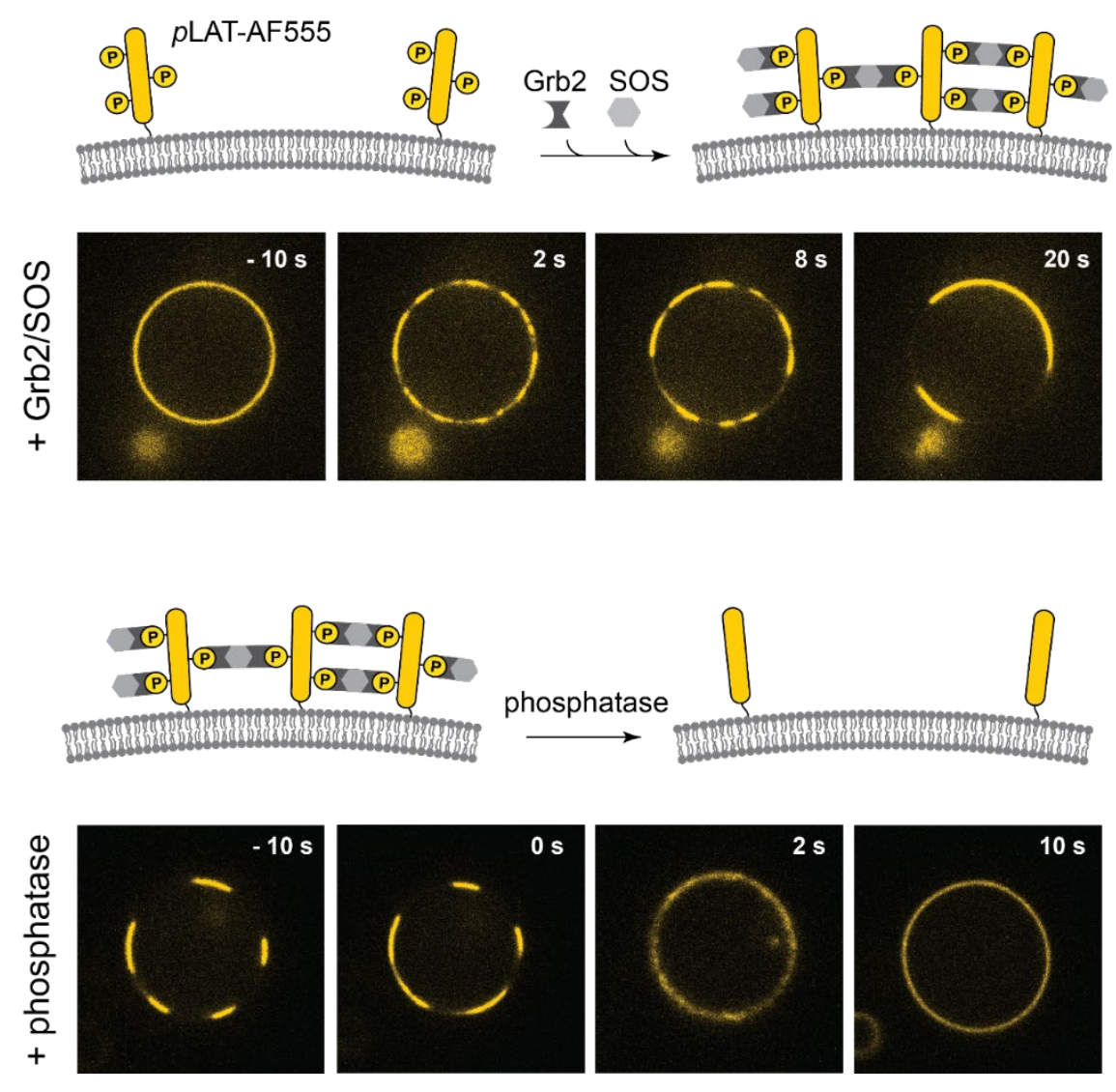

Figure 2. The LAT:Grb2:SOS protein condensate was reconstituted on GUVs. His-tagged phosphor-LAT ( $p$ LAT) is associated with the vesicles by chelating to Ni-NTA lipids. The introduction of Grb2 and SOS results in extended networks of LAT condensate, visualized by the AF555 fluorescence in the confocal microscopy (top). The LAT:Grb2:SOS assembly can be reversed by dephosphorylation of LAT by phosphatase (YopH) (bottom). 


\begin{tabular}{|c|c|c|c|c|c|}
\hline DOPC & $\sum \mathrm{Ni}$-DOGS & TR-DHPE & (๑ $p L A T$ & $\mathbf{x}$ & Grb2-AF647 \\
\hline$\{$ DPPC & I Cholesterol & OG-DHPE & & O & SOSPR $^{P R}$ \\
\hline
\end{tabular}
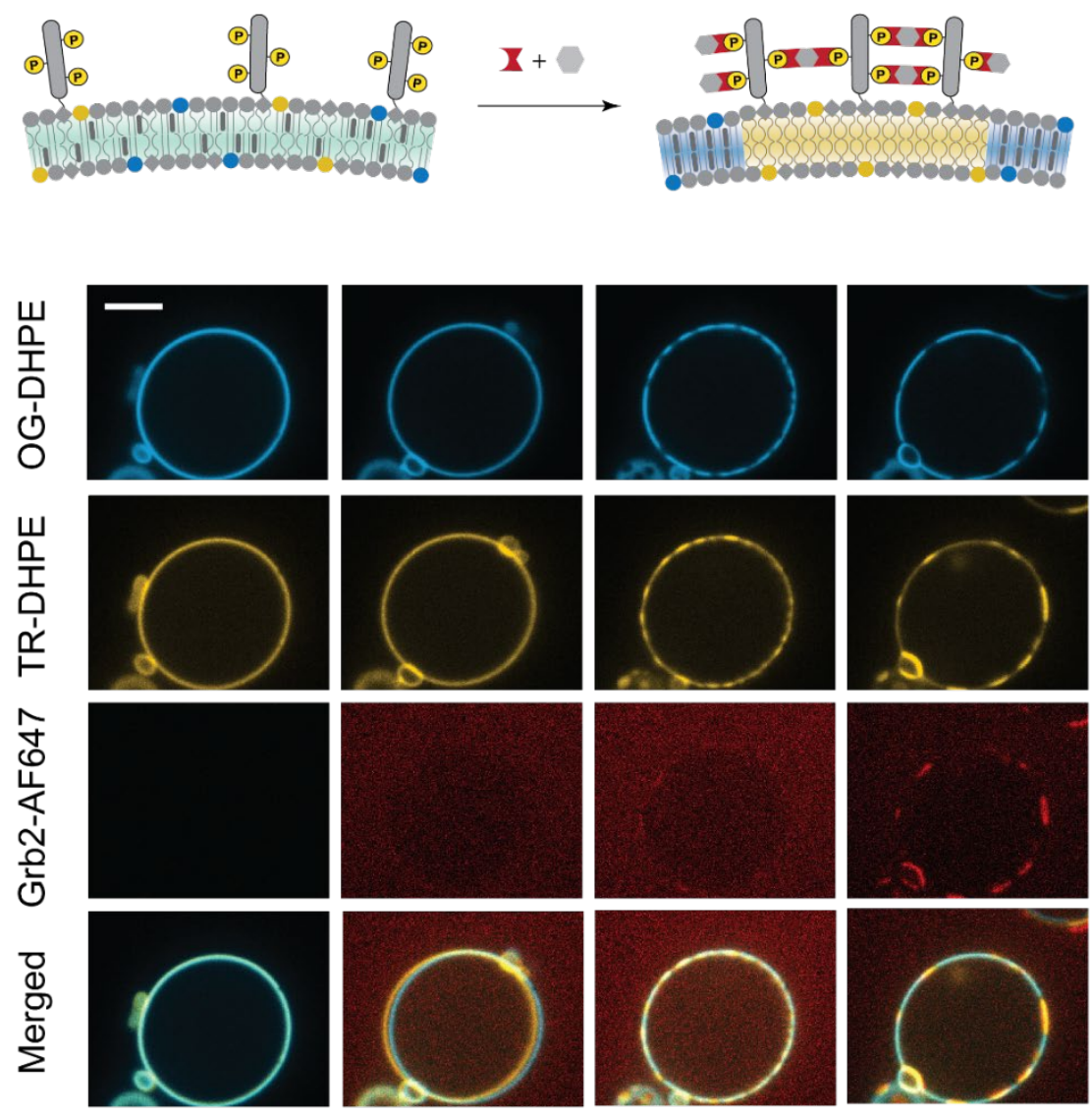

$-10 s$

Os

$6 s$

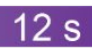

Figure 3. The LAT:Grb2:SOS condensate induces lipid phase separation on GUVs. Starting with a temperature $\left(31^{\circ} \mathrm{C}\right)$ above its $T_{\text {misc }}\left(29^{\circ} \mathrm{C}\right)$, the lipids is spatially homogeneous initially. As the proteins assembled (visualized by Grb2-AF647), the lipids undergo liquid-liquid phase transition. OG-DHPE and TR-DHPE mark the $L_{\mathrm{o}}$ and $L_{\mathrm{d}}$ regions, respectively. 

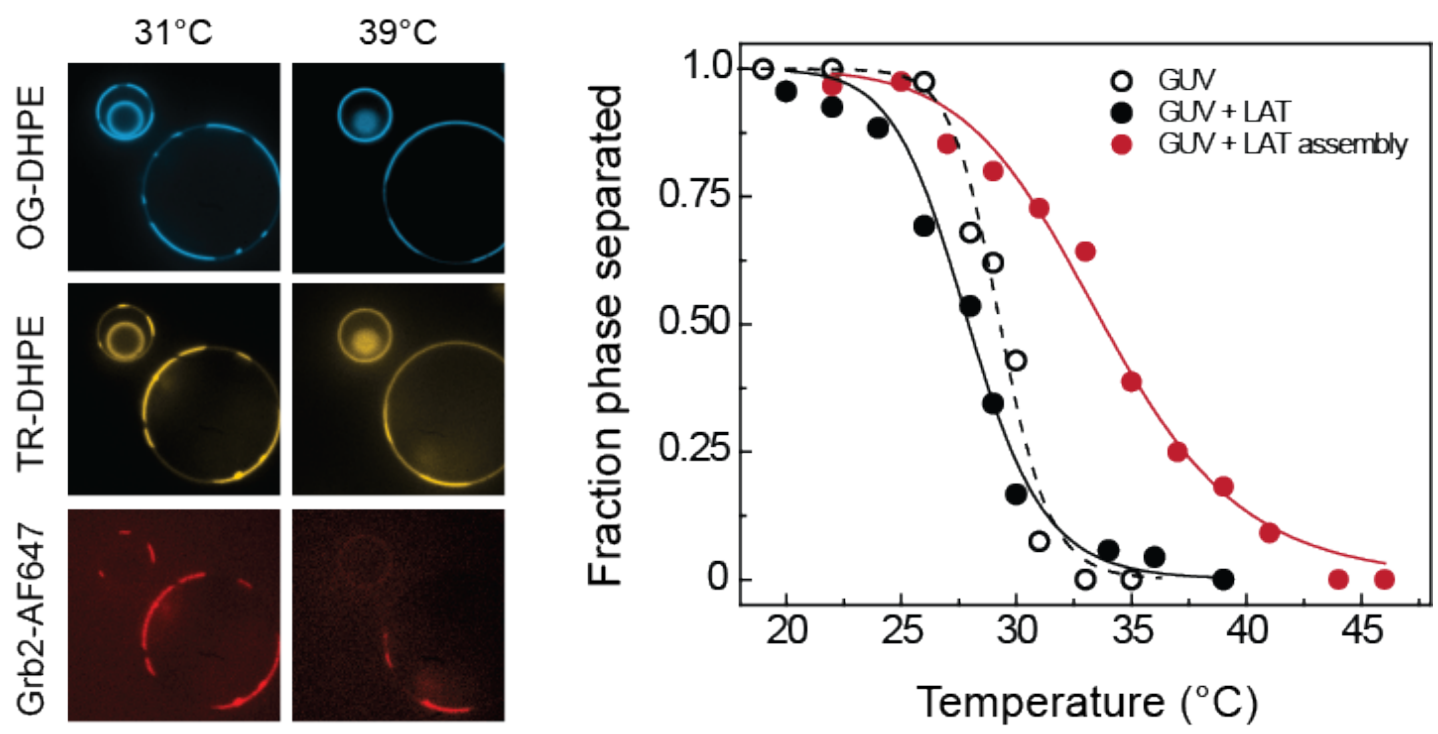

Figure 4. (Left) At $31^{\circ} \mathrm{C}$, the GUVs associated with LAT:Grb2:SOS clusters are phase-separated. Note that the smallest vesicle, invaginated within a larger vesicle, is inaccessible to the proteins, and remains homogeneous. At $39^{\circ} \mathrm{C}$, Grb2 has dissociated from the smaller vesicle, which became homogeneous. For the larger vesicle on which the protein condensate remains, lipid the phase separation also remains. (Right) The miscibility transition temperatures were measured for bare GUVs, GUVs with LAT, and GUVs with the LAT condensate. The difference in Tm between bare GUVs and LAT-associated GUVs are minimal. However, it is increased significantly in presence of the protein assembly. The data primarily reflect temperature-dependent LAT:Grb2:SOS interactions rather than GUV phase separation, as the protein assembly becomes unstable at high temperatures and dissociate from the vesicles. However, hypothetically, stable LAT:Grb2:SOS interactions would further increase the apparent $T_{\text {misc. }}$ 
A
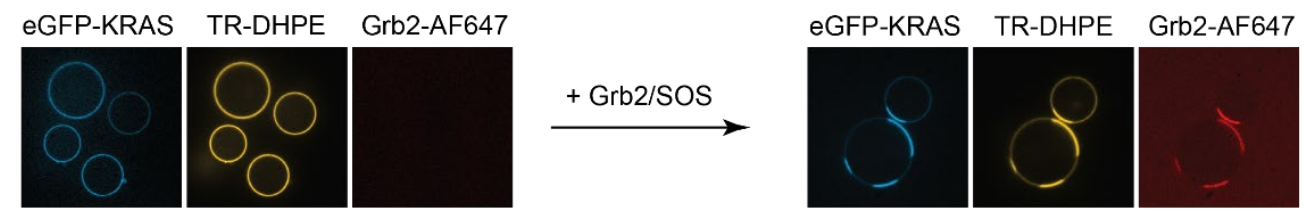

B phosphorylation

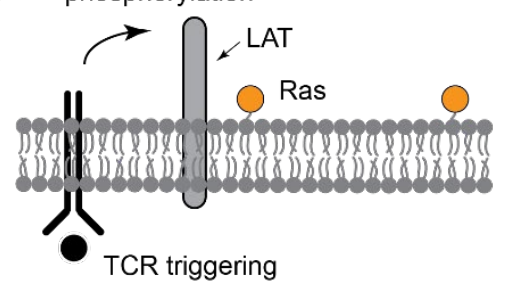

assembly-mediated lipid reorganization

downstream protein reorganization

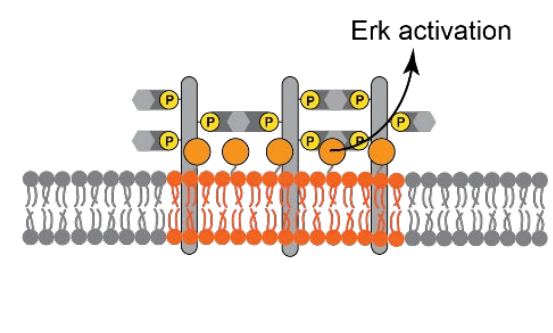

Figure 5. (A) The LAT:Grb2:SOS condensate on GUVs results in segregation of K-Ras into the $L_{\mathrm{d}}$ region with the condensate, suggesting that spatial organization mediated by protein assemblies can propagate downstream of the signaling pathway via lipids. (B) This lipid phase separation induced by protein organization may underlie lipid rafts seen in TCR clusters. 


\section{References}

1. Singer, S. J., and G. L. Nicolson. 1972. The fluid mosaic model of the structure of cell membranes. Science 175:720-731.

2. Shimshick, E. J., and H. M. McConnell. 1973. Lateral phase separation in phospholipid membranes. Biochemistry 12:2351-2360.

3. Brûlet, P., and H. M. McConnell. 1976. Kinetics of phase equilibrium in a binary mixture of phospholipids. J. Am. Chem. Soc. 98:1314-1318.

4. Hong-wei, S., and H. McConnell. 1975. Phase separations in phospholipd membranes. Biochemistry 14:847-854.

5. Luna, E. J., and H. M. McConnell. 1977. Lateral phase separations in binary mixtures of phospholipids having different charges and different crystalline structures. Biochim. Biophys. Acta 470:303-316.

6. Luna, E. J., and H. M. McConnell. 1978. Multiple phase equilibria in binary mixtures of phospholipids. Biochim. Biophys. Acta 509:462-473.

7. Stier, A., and E. Sackmann. 1973. Spin labels as enzyme substrates. Heterogeneous lipid distribution in liver microsomal membranes. Biochim. Biophys. Acta 311:400-408.

8. Simons, K., and E. Ikonen. 1997. Functional rafts in cell membranes. Nature 387:569572.

9. Simons, K., and D. Toomre. 2000. Lipid rafts and signal transduction. Nat. Rev. Mol. Cell Biol. 1:31-39.

10. Simons, K., and M. J. Gerl. 2010. Revitalizing membrane rafts: new tools and insights. Nat. Rev. Mol. Cell Biol. 11:688-699.

11. Munro, S. 2003. Lipid rafts: elusive or illusive? Cell 115:377-388.

12. Nichols, B. 2005. Cell biology: without a raft. Nature 436:638-639.

13. Hancock, J. F. 2006. Lipid rafts: contentious only from simplistic standpoints. Nat. Rev. Mol. Cell Biol. 7:456-462.

14. Levental, I., K. R. Levental, and F. A. Heberle. 2020. Lipid Rafts: Controversies Resolved, Mysteries Remain. Trends Cell Biol. 30:341-353.

15. Baumgart, T., S. T. Hess, and W. W. Webb. 2003. Imaging coexisting fluid domains in biomembrane models coupling curvature and line tension. Nature 425:821-824.

16. Veatch, S. L., and S. L. Keller. 2002. Organization in lipid membranes containing cholesterol. Phys Rev Lett 89:268101.

17. Oglecka, K., P. Rangamani, B. Liedberg, R. S. Kraut, and A. N. Parikh. 2014. Oscillatory phase separation in giant lipid vesicles induced by transmembrane osmotic differentials. eLife 3:e03695.

18. Leslie, M. 2011. Mysteries of the cell. Do lipid rafts exist? Science 334:1046-1047.

19. Sevcsik, E., M. Brameshuber, M. Fölser, J. Weghuber, A. Honigmann, and G. J. Schütz. 2015. GPI-anchored proteins do not reside in ordered domains in the live cell plasma membrane. Nat Commun 6:6969.

20. Lee, I. H., S. Saha, A. Polley, H. Huang, S. Mayor, M. Rao, and J. T. Groves. 2015. Live cell plasma membranes do not exhibit a miscibility phase transition over a wide range of temperatures. J. Phys. Chem. B 119:4450-4459. 
21. Saha, S., I. H. Lee, A. Polley, J. T. Groves, M. Rao, and S. Mayor. 2015. Diffusion of GPI-anchored proteins is influenced by the activity of dynamic cortical actin. Mol Biol Cell 26:4033-4045.

22. Rayermann, S. P., G. E. Rayermann, C. E. Cornell, A. J. Merz, and S. L. Keller. 2017. Hallmarks of Reversible Separation of Living, Unperturbed Cell Membranes into Two Liquid Phases. Biophys. J. 113:2425-2432.

23. Veatch, S. L., P. Cicuta, P. Sengupta, A. Honerkamp-Smith, D. Holowka, and B. Baird. 2008. Critical fluctuations in plasma membrane vesicles. ACS Chem Biol 3:287-293.

24. Rao, M., and S. Mayor. 2014. Active organization of membrane constituents in living cells. Curr. Opin. Cell Biol. 29:126-132.

25. Stone, M. B., S. A. Shelby, M. F. Núñez, K. Wisser, and S. L. Veatch. 2017. Protein sorting by lipid phase-like domains supports emergent signaling function in B lymphocyte plasma membranes. eLife 6:e19891.

26. Monks, C. R., B. A. Freiberg, H. Kupfer, N. Sciaky, and A. Kupfer. 1998. Threedimensional segregation of supramolecular activation clusters in T cells. Nature 395:8286.

27. Dustin, M. L., and J. A. Cooper. 2000. The immunological synapse and the actin cytoskeleton: molecular hardware for T cell signaling. Nat. Immunol. 1:23-29.

28. Yokosuka, T., K. Sakata-Sogawa, W. Kobayashi, M. Hiroshima, A. Hashimoto-Tane, M. Tokunaga, M. L. Dustin, and T. Saito. 2005. Newly generated T cell receptor microclusters initiate and sustain T cell activation by recruitment of Zap70 and SLP-76. Nat. Immunol. 6:1253-1262.

29. Rapp, M., E. Granseth, S. Seppälä, and G. von Heijne. 2006. Identification and evolution of dual-topology membrane proteins. Nat. Struct. Mol. Biol. 13:112-116.

30. Lillemeier, B. F., M. A. Mörtelmaier, M. B. Forstner, J. B. Huppa, J. T. Groves, and M. M. Davis. 2010. TCR and Lat are expressed on separate protein islands on T cell membranes and concatenate during activation. Nat. Immunol. 11:90-96.

31. Dustin, M. L., and J. T. Groves. 2012. Receptor signaling clusters in the immune synapse. Annu Rev Biophys 41:543-556.

32. Yi, J., L. Balagopalan, T. Nguyen, K. M. McIntire, and L. E. Samelson. 2019. TCR microclusters form spatially segregated domains and sequentially assemble in calciumdependent kinetic steps. Nat Commun 10:277.

33. Cerny, J., H. Stockinger, and V. Horejsi. 1996. Noncovalent associations of T lymphocyte surface proteins. Eur. J. Immunol. 26:2335-2343.

34. Janes, P. W., S. C. Ley, A. I. Magee, and P. S. Kabouridis. 2000. The role of lipid rafts in T cell antigen receptor (TCR) signalling. Semin. Immunol. 12:23-34.

35. Drevot, P., C. Langlet, X. J. Guo, A. M. Bernard, O. Colard, J. P. Chauvin, R. Lasserre, and H. T. He. 2002. TCR signal initiation machinery is pre-assembled and activated in a subset of membrane rafts. EMBO J. 21:1899-1908.

36. Douglass, A. D., and R. D. Vale. 2005. Single-molecule microscopy reveals plasma membrane microdomains created by protein-protein networks that exclude or trap signaling molecules in T cells. Cell 121:937-950.

37. Glebov, O. O., and B. J. Nichols. 2004. Lipid raft proteins have a random distribution during localized activation of the T-cell receptor. Nat. Cell Biol. 6:238-243. 
38. Bunnell, S. C., D. I. Hong, J. R. Kardon, T. Yamazaki, C. J. McGlade, V. A. Barr, and L. E. Samelson. 2002. T cell receptor ligation induces the formation of dynamically regulated signaling assemblies. J Cell Biol 158:1263-1275.

39. Purcell, A. W., N. P. Croft, and D. C. Tscharke. 2016. Immunology by numbers: quantitation of antigen presentation completes the quantitative milieu of systems immunology! Curr. Opin. Immunol. 40:88-95.

40. Lin, J. J., G. P. O'Donoghue, K. B. Wilhelm, M. P. Coyle, S. T. Low-Nam, N. C. Fay, K. N. Alfieri, and J. T. Groves. 2020. Membrane Association Transforms an Inert AntiTCRbeta Fab' Ligand into a Potent T Cell Receptor Agonist. Biophys. J. 118:2879-2893.

41. Brameshuber, M., F. Kellner, B. K. Rossboth, H. Ta, K. Alge, E. Sevcsik, J. Gohring, M. Axmann, F. Baumgart, N. R. J. Gascoigne, S. J. Davis, H. Stockinger, G. J. Schutz, and J. B. Huppa. 2018. Monomeric TCRs drive T cell antigen recognition. Nat. Immunol. 19:487-496.

42. Rossboth, B., A. M. Arnold, H. Ta, R. Platzer, F. Kellner, J. B. Huppa, M. Brameshuber, F. Baumgart, and G. J. Schutz. 2018. TCRs are randomly distributed on the plasma membrane of resting antigen-experienced T cells. Nat. Immunol. 19:821-827.

43. O'Donoghue, G. P., R. M. Pielak, A. A. Smoligovets, J. J. Lin, and J. T. Groves. 2013. Direct single molecule measurement of TCR triggering by agonist pMHC in living primary T cells. eLife 2:e00778.

44. Lin, J. J. Y., S. T. Low-Nam, K. N. Alfieri, D. B. McAffee, N. C. Fay, and J. T. Groves. 2019. Mapping the stochastic sequence of individual ligand-receptor binding events to cellular activation: T cells act on the rare events. Science Signaling 12:eaat8715.

45. Shin, Y., and C. P. Brangwynne. 2017. Liquid phase condensation in cell physiology and disease. Science 357:eaaf4382.

46. Banani, S. F., H. O. Lee, A. A. Hyman, and M. K. Rosen. 2017. Biomolecular condensates: organizers of cellular biochemistry. Nat Rev Mol Cell Biol 18:285-298.

47. Li, P., S. Banjade, H. C. Cheng, S. Kim, B. Chen, L. Guo, M. Llaguno, J. V. Hollingsworth, D. S. King, S. F. Banani, P. S. Russo, Q. X. Jiang, B. T. Nixon, and M. K. Rosen. 2012. Phase transitions in the assembly of multivalent signalling proteins. Nature 483:336-340.

48. Su, X., J. A. Ditlev, E. Hui, W. Xing, S. Banjade, J. Okrut, D. S. King, J. Taunton, M. K. Rosen, and R. D. Vale. 2016. Phase separation of signaling molecules promotes T cell receptor signal transduction. Science 352:595-599.

49. Huang, W. Y., Q. Yan, W. C. Lin, J. K. Chung, S. D. Hansen, S. M. Christensen, H. L. Tu, J. Kuriyan, and J. T. Groves. 2016. Phosphotyrosine-mediated LAT assembly on membranes drives kinetic bifurcation in recruitment dynamics of the Ras activator SOS. Proc Natl Acad Sci U S A 113:8218-8223.

50. Case, L. B., J. A. Ditlev, and M. K. Rosen. 2019. Regulation of Transmembrane Signaling by Phase Separation. Annu. Rev. Biophys. 48:465-494.

51. Banjade, S., and M. K. Rosen. 2014. Phase Transitions of Multivalent Proteins Can Promote Clustering of Membrane Receptors. eLife 3:e04123.

52. Brangwynne, C. P., C. R. Eckmann, D. S. Courson, A. Rybarska, C. Hoege, J. Gharakhani, F. Julicher, and A. A. Hyman. 2009. Germline P Granules Are Liquid Droplets That Localize by Controlled Dissolution/Condensation. Science 324:1729-1732.

53. Hnisz, D., K. Shrinivas, R. A. Young, A. K. Chakraborty, and P. A. Sharp. 2017. A Phase Separation Model for Transcriptional Control. Cell 169:13-23. 
54. Huang, W. Y. C., S. Alvarez, Y. Kondo, Y. K. Lee, J. K. Chung, H. Y. M. Lam, K. H. Biswas, J. Kuriyan, and J. T. Groves. 2019. A molecular assembly phase transition and kinetic proofreading modulate Ras activation by SOS. Science 363:1098-1103.

55. Huang, W. Y. C., H. K. Chiang, and J. T. Groves. 2017. Dynamic Scaling Analysis of Molecular Motion within the LAT:Grb2:SOS Protein Network on Membranes. Biophys J 113:1807-1813.

56. Houtman, J. C., Y. Higashimoto, N. Dimasi, S. Cho, H. Yamaguchi, B. Bowden, C. Regan, E. L. Malchiodi, R. Mariuzza, P. Schuck, E. Appella, and L. E. Samelson. 2004. Binding specificity of multiprotein signaling complexes is determined by both cooperative interactions and affinity preferences. Biochemistry 43:4170-4178.

57. Houtman, J. C., H. Yamaguchi, M. Barda-Saad, A. Braiman, B. Bowden, E. Appella, P. Schuck, and L. E. Samelson. 2006. Oligomerization of signaling complexes by the multipoint binding of GRB2 to both LAT and SOS1. Nat. Struct. Mol. Biol. 13:798-805.

58. Kortum, R. L., L. Balagopalan, C. P. Alexander, J. Garcia, J. M. Pinski, R. K. Merrill, P. H. Nguyen, W. Li, I. Agarwal, I. O. Akpan, C. L. Sommers, and L. E. Samelson. 2013. The ability of Sos1 to oligomerize the adaptor protein LAT is separable from its guanine nucleotide exchange activity in vivo. Sci Signal 6:ra99.

59. Balagopalan, L., R. L. Kortum, N. P. Coussens, V. A. Barr, and L. E. Samelson. 2015. The Linker for Activation of T Cells (LAT) Signaling Hub: From Signaling Complexes to Microclusters. J. Biol. Chem. 290:26422-26429.

60. Zhu, M., E. Janssen, and W. Zhang. 2003. Minimal requirement of tyrosine residues of linker for activation of T cells in TCR signaling and thymocyte development. J Immunol 170:325-333.

61. Lin, J., and A. Weiss. 2001. Identification of the minimal tyrosine residues required for linker for activation of T cell function. J Biol Chem 276:29588-29595.

62. Veatch, S. L., and S. L. Keller. 2003. Separation of liquid phases in giant vesicles of ternary mixtures of phospholipids and cholesterol. Biophys J 85:3074-3083.

63. Veatch, S. L., I. V. Polozov, K. Gawrisch, and S. L. Keller. 2004. Liquid domains in vesicles investigated by NMR and fluorescence microscopy. Biophys. J. 86:2910-2922.

64. Ellis, C. A., and G. Clark. 2000. The importance of being K-Ras. Cell. Signal. 12:425434.

65. Friday, B. B., and A. A. Adjei. 2005. K-ras as a target for cancer therapy. Biochim. Biophys. Acta 1756:127-144.

66. Simanshu, D. K., D. V. Nissley, and F. McCormick. 2017. RAS Proteins and Their Regulators in Human Disease. Cell 170:17-33.

67. Weise, K., S. Kapoor, C. Denter, J. Nikolaus, N. Opitz, S. Koch, G. Triola, A. Herrmann, H. Waldmann, and R. Winter. 2011. Membrane-mediated induction and sorting of K-Ras microdomain signaling platforms. J. Am. Chem. Soc. 133:880-887.

68. Gillette, W. K., D. Esposito, M. Abreu Blanco, P. Alexander, L. Bindu, C. Bittner, O. Chertov, P. H. Frank, C. Grose, J. E. Jones, Z. Meng, S. Perkins, Q. Van, R. Ghirlando, M. Fivash, D. V. Nissley, F. McCormick, M. Holderfield, and A. G. Stephen. 2015. Farnesylated and methylated KRAS4b: high yield production of protein suitable for biophysical studies of prenylated protein-lipid interactions. Sci Rep 5:15916.

69. Chung, J. K., Y. K. Lee, J. P. Denson, W. K. Gillette, S. Alvarez, A. G. Stephen, and J. T. Groves. 2018. K-Ras4B Remains Monomeric on Membranes over a Wide Range of Surface Densities and Lipid Compositions. Biophys J 114:137-145. 
70. Hancock, J. F., H. Paterson, and C. J. Marshall. 1990. A polybasic domain or palmitoylation is required in addition to the CAAX motif to localize $\mathrm{p} 21$ ras to the plasma membrane. Cell 63:133-139.

71. Cadwallader, K. A., H. Paterson, S. G. Macdonald, and J. F. Hancock. 1994. Nterminally myristoylated Ras proteins require palmitoylation or a polybasic domain for plasma membrane localization. Mol. Cell. Biol. 14:4722-4730.

72. Liu, A. P., and D. A. Fletcher. 2006. Actin polymerization serves as a membrane domain switch in model lipid bilayers. Biophys. J. 91:4064-4070.

73. Honigmann, A., S. Sadeghi, J. Keller, S. W. Hell, C. Eggeling, and R. Vink. 2014. A lipid bound actin meshwork organizes liquid phase separation in model membranes. Elife 3:e01671.

74. Hammond, A. T., F. A. Heberle, T. Baumgart, D. Holowka, B. Baird, and G. W. Feigenson. 2005. Crosslinking a lipid raft component triggers liquid ordered-liquid disordered phase separation in model plasma membranes. Proc Natl Acad Sci U S A 102:6320-6325.

75. Zhang, W., J. Sloan-Lancaster, J. Kitchen, R. P. Trible, and L. E. Samelson. 1998. LAT: the ZAP-70 tyrosine kinase substrate that links T cell receptor to cellular activation. Cell 92:83-92.

76. Shah, N. H., Q. Wang, Q. R. Yan, D. Karandur, T. A. Kadlecek, I. R. Fallahee, W. P. Russ, R. Ranganathan, A. Weiss, and J. Kuriyan. 2016. An electrostatic selection mechanism controls sequential kinase signaling downstream of the T cell receptor. eLife 5:e20105.

77. Kaizuka, Y., and J. T. Groves. 2004. Structure and dynamics of supported intermembrane junctions. Biophys. J. 86:905-912.

78. Veatch, S. L., and S. L. Keller. 2003. Separation of liquid phases in giant vesicles of ternary mixtures of phospholipids and cholesterol. Biophys. J. 85:3074-3083.

79. Parthasarathy, R., C. H. Yu, and J. T. Groves. 2006. Curvature-modulated phase separation in lipid bilayer membranes. Langmuir 22:5095-5099.

80. Hansen, S. D., W. Y. C. Huang, Y. K. Lee, P. Bieling, S. M. Christensen, and J. T. Groves. 2019. Stochastic geometry sensing and polarization in a lipid kinase-phosphatase competitive reaction. Proc. Natl. Acad. Sci. USA 116:15013-15022.

81. Lee, A. A., W. Y. C. Huang, S. D. Hansen, N. H. Kim, S. Alvarez, and J. T. Groves. Stochasticity and positive feedback enable enzyme kinetics at the membrane to sense reaction size. Submitted.

82. Courtney, A. H., W. L. Lo, and A. Weiss. 2018. TCR Signaling: Mechanisms of Initiation and Propagation. Trends Biochem Sci 43:108-123.

83. Abraham, R. T., and A. Weiss. 2004. Jurkat T cells and development of the T-cell receptor signalling paradigm. Nat. Rev. Immunol. 4:301-308. 